\title{
Hygiene Facilities and Practices for Vended Meats at Selected Highway Markets in Uganda
}

\author{
Ananias Bagumire ${ }^{1}$, Karumuna Rollanda ${ }^{2, *}$ \\ ${ }^{1}$ National Food Safety Foundation (NFSF), the Affiliated Institution of the Food Safety Associates Limited, Kampala, Uganda \\ ${ }^{2}$ Department of Environment Management, College of Agriculture and Environmental Sciences, Makerere University, Kampala, Uganda
}

\section{Email address:}

karumunaroland@gmail.com (K. Rollanda)

${ }^{*}$ Corresponding author

\section{To cite this article:}

Ananias Bagumire, Karumuna Rollanda. Hygiene Facilities and Practices for Vended Meats at Selected Highway Markets in Uganda. Journal of Food and Nutrition Sciences. Vol. 5, No. 1, 2017, pp. 1-10. doi: 10.11648/j.jfns.20170501.11

Received: December 17, 2016; Accepted: January 3, 2017; Published: January 24, 2017

\begin{abstract}
A study on hygiene facilities and practices for vendors of ready-to-eat roasted meat at selected highway markets was conducted. The objective of the study was to compare the current hygiene status with recommended best practices. Recommended conditions for hygiene facilities such as hand washing items, food storage equipments, food preparation and processing equipments as well as display and service facilities were assessed at 41 stalls using checklists. Questionnaires covering meat purchase and delivery, storage, preparation, processing, display and service were also administered to 180 vendors to assess their hygiene practices. A scale of 0-4 was used to assess the conformity of hygiene facilities. The majority $(63 \%)$ of the hygiene facilities assessed scored below 2 denoting low conformity to recommended conditions. Hand washing facilities were the most deficient with a score of 0.34 . Fair scores were obtained for aprons (2.5). The low scores obtained during the assessment of hygiene facilities concurred with the results obtained from the assessment of hygiene practices. The majority $(71.5 \%)$ of the responses from vendors showed poor hygiene practices. Therefore, the hygiene facilities and practices did not conform to recommended best conditions. This means that there is a high risk of contamination of ready-to-eat roasted meat in the highway markets due to poor hygiene. Improved hygiene facilities, training and awareness creation among vendors and market authorities are required to ensure safety of ready-to-eat meats.
\end{abstract}

Keywords: Highway Markets, Hygiene, Ready-to-Eat Food, Conformity, Best Practices

\section{Introduction}

Appropriate hygiene facilities and practices have become an integral part of the food safety management strategies for food handling operations which may include food vending markets. Hygiene is a set of practices or conditions performed or put in place for the preservation and or maintenance of health and preventing disease and or promoting health living. Food hygiene involves practices that prevent food poisoning such as prevention of contamination of food with pathogens, separating raw and cooked foods, processing foods for the appropriate time and temperature, storage of food at the proper temperature and use of safe water and raw materials $[1,2]$.

Hygiene in food handling areas such as highway food vending markets is facilitated by hand washing facilities and items, food storage equipments such as cupboards, boxes, bags, refrigerators and buckets. Hygiene is also facilitated by food processing and preparation facilities and items such as roasting stoves, roasting wire-meshes, roasting sticks and cutting/chopping facilities/items such as knives, machetes/pangas and chopping woods/boards [3]. Hygiene during display and service of ready-to-eat foods is facilitated by display platforms, dressing items such as aprons, mouth, hair and beard restraints/covers and packing materials such as plastic bags and wrappers or packages. It is also influenced by the cleaning methods deployed, cleaning schedules/frequency, the materials making food contact surfaces as well as any other items and facilities that come in contact with food [4].

The highway food vending markets especially those that deal in ready-to-eat food feed thousands of people daily with a wide variety of foods that are relatively cheap and easily accessible and therefore plays an important role in meeting the food 
demands of the travelers. This form of food trade involves venders preparing food at strategic roadside points where vehicles with travelers stop [5]. The vending of food involves selling of ready-to-eat foods which is either served on roasting sticks or in containers to travelers in vehicles that stop by the road side at the highway market points. The food products sold include meats such as chicken, beef and goat meat. Other readyto-eat food products sold include cassava, potatoes, plantain "gonja", chapattis (unleavened flat bread that originates from India and other oriental countries), fruits, vegetables, drinks such as water, soda and fruit juices. The trade is mostly run by young and energetic men and women and therefore is considered key for employment of youth and women.

The hygiene practices in food vending markets in Uganda are poor which raises fears of some consumers that they may compromise the safety of the food that is vended [3]. Reports indicate that most food vendors in Uganda do not purchase their foods from licensed or reliable suppliers. They handle food with bare hands; their cutleries are dirty; they stack ready-to-eat food one on top of the other during display, storing or serving; they do not use food grade packing materials; they blow air into packing materials; handle money alongside food service/selling; do not process or display the food to/at the recommended temperature; they also do not wear protective gear during food handling and some keep long nails, wear jewelry and continue working even when they are not in good health $[1,3]$.

The hygiene facilities and practices in highway markets may not be an exception. The poor hygiene facilities and practices in highway food markets have potential to contaminate the food leading to the outbreak of food borne illnesses especially gastrointestinal infectious diseases such as gastroenteritis, dysentery, brucellosis, anthrax, botulism, emetic intoxication, pharyngitis, nephritic sequelae, sore throat, listeriosis (various manifestations), tuberculosis, Q fever and enteric infection [6]. These illnesses may result into permanent body damage, exorbitant expenditure to mitigate the illnesses or even death. The contamination of ready-to-eat meat products due to poor hygiene has the potential effect of raising fear for the safety of consumers. This would discourage some customers who may worry about the effects such foods may have on their health, leading to reduced sales and income of vendors and all the individuals that are involved in the value/supply chain. This will spill into wider effects on local and national revenue collection. This study was therefore aimed to establish the extent of hygiene and how this could affect the safety of readyto-eat meats that are sold in highway markets.

\section{Materials and Methods}

\subsection{Selection of Study Sites}

Two highway markets (Lukaya and Najembe) were selected for this study. The two are among the major highway markets found on major roads leading out of Kampala city. Najembe market is located approximately $45 \mathrm{~km}$ from Kampala city on Jinja highway in Buikwe district heading to the eastern part of Uganda. Lukaya market is located approximately $100 \mathrm{~km}$ on Kampala-Masaka highway in Kalungu district heading to the southern and western parts of Uganda. These markets were also selected for the study because they had a large population of food vendors when compared to other highway markets.

\subsection{Nature, Organisation and Business in the Markets}

Najembe and Lukaya markets have populations of about 320-350 vendors and 350-400 vendors respectively, who are involved in the sale of various foods. Their main activities include mainly roasting and selling of ready-to-eat food stuffs to travellers both leaving and coming out of Kampala city. The food products sold include meats such as chicken, beef and goat meat. Other ready-to-eat products sold include cassava, potatoes, plantain "gonja", chapattis, fruits, vegetables and drinks such as water, soda and fruit juice.

The purchase and delivery of raw food stuffs by vendors is done in such a way that each vendor buys his/her products for sale separately from different or same suppliers. Deliveries are therefore done on individual-to-individual basis and each vendor finds a way of storing, preparing, processing and displaying products on their own. The vendor's stall serves as a delivery, storage, preparation, processing and display point. The markets therefore do not have designated delivery, storage, preparation, processing and display sections. At Najembe market over 90-110 chicken, 1-2 cows and 5-8 goats are slaughtered; while over 80-100 chicken, 2-3 cows and 7-10 goats are slaughtered at Lukaya market. All together; vendors in the two markets sell to over 500 travellers who buy meats at Najembe or Lukaya markets daily. The remainder of the meat is sold to the communities around and/or to fellow vendors. The markets are led by chairpersons and their committee members.

\subsection{Selection of Products Studied}

The study concentrated on high risk ready-to-eat food products that are sold in highway markets. The identification of high risk products was based on the U. S. Food and Drug Administration (FDA) guidelines for identifying high risk foods [7]. The types of foods vended in the two markets that are more likely to be implicated as vehicles of food poisoning organisms and lead to gastrointestinal infections (illness) were included for the study. These types of food are normally high in protein and require strict temperature control and protection from contamination [7]. They included roasted meat and poultry products such as beef, pork, lamb, chicken, turkey and duck. The study concentrated on highway roasted meats of chicken, beef and goat meat. These products are very popular among travelers in Uganda and are mainly sold as roasted ready-to-eat products.

\subsection{Selection of the Vendors and Stalls for the Study}

\subsubsection{Selection of Stalls Used in the Assessment of Hygiene Facilities}

A total of 41 stalls of chicken, beef and goat meat were 
selected from Lukaya and Najembe markets. The stalls are raised using wooden poles and roofed with either iron sheets or grass; some have short walls that are raised to about 2 metres above the ground (window level) and their floors are either made of soil or rough concrete. In Najembe market, 9 stalls for chicken, 7 stalls for goat meat and 5 stalls for beef were selected (total 21). In Lukaya, 7 stalls for chicken, 5 stalls for goat meat and 8 stalls for beef were selected (total $20)$. The 41 stalls were arrived at after conducting an assessment of stalls for ownership and the mode of operation. The assessment was conducted by holding meetings with the leadership of the markets in which the chairpersons assisted in identifying the owners of the stalls. This was followed by inquiries among the owners (food vendors) about the mode of operation of stalls.

In the assessment, it was found that Najembe market had 6 housing structures that accommodated all stalls for chicken, goat meat and beef vendors; while Lukaya market had 3 housing structures that accommodated all the stalls for chicken, goat meat and beef vendors. Each stall within the housing structures was shared by at least 4-8 vendors in Najembe and 5-9 vendors in Lukaya markets. The vendors that operated these stalls roasted their meat at different times of the day.

\subsubsection{Selection of Vendors Used to Assess the Status of Hygiene Practices}

After assessing the ownership and mode of operation in the markets, 30 vendors each for chicken, goat meat and beef were selected randomly from each market to respond to the prepared set of questions in the questionnaire all totalling to 90 vendors who responded to the questions in each market. A total of 180 vendors were therefore selected from the two markets. Only vendors, whose stalls were assessed for hygiene facilities, were selected for this interview.

\subsection{Research Instruments and Tools}

\subsubsection{Development of the Checklists for Assessing the Hygiene Facilities}

The development of the checklist that was used to assess the hygiene facilities was undertaken by first reviewing the recommended conditions relevant to hygiene facilities of highway markets and adopting the relevant sections of the FAO/WHO Codex Alimentarius international standards which relate to hygiene facilities of food vending markets. Specifically the FAO/WHO regional guidelines for the design of control measures for street-vended foods (Africa) of 1997 were amended as appropriate and used to benchmark the hygiene facilities in the highway markets in Uganda. Uganda's Food and Drug Act (1964)-part II (Offences in connection with preparation and sale of injurious foods, general protection for purchasers of food and offence to sell of food unfit for human consumption) and Public Health Act-1969 (part XIIprotection of food stuffs) were also respectively reviewed and relevant provisions that are aligned to the modern food hygiene best facilities were incorporated in the checklist. Also incorporated in the checklists were relevant sections in the manual of standard operating procedures for fish inspection and quality assurance [8].

The checklist was formulated with four (4) detailed sections to assess the hygiene facilities of individual stalls found in the studied highway markets. The sections covered (1) conformity to recommended conditions of hand washing facilities, (2) conformity to recommended conditions of food storage equipments, (3) conformity to recommended conditions of food preparation and processing equipments at the stall and (4) conformity to recommended conditions of display and sell of food. All these conditions were observed for presence or absence on the individual vendor's stalls during field work exercise.

Specific recommended conditions for best hygiene facilities under each of the particular requirements or elements were included in the checklists. In developing the checklist, the format and approach used by Bagumire et al. [9] was adapted with some modifications.

\subsubsection{Questionnaire for Assessing the Status of Hygiene Practices}

Seven (7) detailed sections with a total of 18 questions were formulated each comprised of specific hygiene practices to be assessed. The ideal hygiene practices on which questions are based were adapted from FAO/WHO Codex Alimentarius Commission [10], Uganda's Food and Drug Act [11], Public Health Act [12] and MAAIF SOPs for fish inspection and quality assurance [8]. The questionnaire included a bio data section and vendors' background section covering vendor's age, sex, level of education, years spent in food vending, products vended and nature of customers.

The other sections investigated the hygiene challenges faced by vendors and actions required to overcome them; sources of products prepared by vendors and hygiene conditions of food at the time of delivery and during storage. The questionnaire also had sections on food preparation; the categories of food prepared and the means used to prevent contamination during food preparation including storage of personal belongings; the foods that are roasted and the means used to prevent food contamination including temperature range control and temperature of food during display and service as well as the means of preserving the food during display. The questionnaire further investigated how the vendors ensured personal hygiene during food handling. Also included in the questionnaire was a section that investigated whether vendors had training on hygiene related issues.

\subsection{Assessment of the Hygiene Facilities and Practices}

An inspection approach for food markets was adapted to carry out a study of hygiene facilities in the selected highway markets. Prior to the study of hygiene facilities in selected highway markets, reconnaissance visits were undertaken to Najembe and Lukaya highway markets. Contact persons were identified and field data collection schedules arranged with market authorities during the reconnaissance visits. Among the key contacts used were the chairpersons of the market vendors, the local health authorities and security personnel. During the scheduled field visits, meetings were first held with the chairpersons of the 
market vendors where protocols for collecting the data were agreed upon. A guided tour around the market was conducted and hygiene facilities at the food vendors' stalls and shared facilities were examined for chicken, beef and goat meat vendors through observation. The checklists were used to assess the facilities by ticking the elements of the recommended conditions in the checklist that were observed to be complied with by the stalls or shared facilities.

After assessing the facilities, interviews were held with vendors to establish the hygiene practices undertaken by vendors using questionnaires. A meeting with vendors was held at the end of the assessment and interviews where an indepth discussion was undertaken to clarify some of the contentious observations and responses received. Each field visit ended with a meeting with market authorities to verify the information obtained from the food vendors.

\subsection{Data Analysis}

\subsubsection{Data from Checklists}

The scale of scores from 0 to 4 where zero denoted nonconformity to recommended conditions while 4 denoted high level conformity to recommended conditions was developed. The score scale had intermediary levels of conformity representing low conformity (1), fair conformity (2) and good conformity (3).

The requirements ticked during assessment of facilities were first denoted by 1 score while the requirements not ticked were denoted by 0 score. All elements used to evaluate conformity with recommended conditions in 41 checklists that were used at the stalls were allocated 1 or zero depending on the observations made. The hygiene requirements/elements in the checklists that were ticked were then counted and the number of requirements/elements ticked was then expressed as a fraction out of the total number of requirements/elements (for each facility) investigated as was included in the checklist. The generated fraction was then multiplied by 4 the score given to conditions for full compliance (conformity). For example if a facility had 10 requirements/elements to be investigated in the checklist and had scored $6 / 10$ requirements then this fraction would be multiplied by $4($ eg. $6 / 10 \times 4)$ since 4 was the score that denoted full compliance. The result of the calculation became the final score for a particular facility.

The method used to calculate final scores is expressed in formula (1):

$\frac{\text { Number of requirements/elements ticked as conforming to required conditions }}{\text { Total number of recommended conditions for the facility investigated }} \times 4$ (the highest level of conformity)

Final scores for each requirement and facility were sorted, coded and entered in the computer statistical package for social scientists (SPSS) where high level of conformity was coded 4 , good conformity was coded 3 , fair conformity was coded 2 , low conformity was coded 1 and non-conformity was coded 0 . Then using descriptive statistics percentages (\%) of scores for each of the conditions investigated in the two markets were generated, tabulated and compared as appropriate.

\subsubsection{Data from Questionnaires}

A coding list was generated which was then used to enter all the responses given in the questionnaires. The coding process involved examining the raw qualitative data in the questionnaires and discussion sheets which was in the form of words, phrases, sentences or paragraphs and assigning them CODES or labels. For example if a question had options $\mathrm{a}, \mathrm{b}, \mathrm{c}, \mathrm{d}$ and $\mathrm{e}$ the following codes were given, $\mathrm{a}=1$, $b=2, c=3, d=4$ and $e=5$. The codes given were entered in SPSS to represent the responses obtained from the food vendors of Lukaya and Najembe markets. Descriptive statistics were used to generate frequencies and percentages.

\section{Results and Discussion}

\subsection{Conformity to Recommended Conditions of Hand Washing Facilities}

Overall, 94\% of the stalls assessed for conformity to recommended conditions of hand washing facilities at Najembe and Lukaya markets scored 0 denoting nonconformity. Two percent $(2 \%)$ of the stalls in both markets scored 1 denoting low conformity to recommended conditions, $3 \%$ scored 2 , denoting a fair conformity while $1 \%$ scored 3 denoting good conformity. None of the facilities scored 4 (Table 1). The non-conformity to recommended conditions of hand washing facilities that was observed in almost all the stalls was because meat vendors did not have hand washing facilities at their stalls. The majority of the vendors interviewed $(91 \%)$ were not aware that they needed to wash their hands regularly and therefore did not wash hands to the required standard during food handling. This points to the lack of awareness and sensitization about the need for hand washing. Lack of knowledge about the need for frequent hand washing is attributed to lack of food hygiene training. This was confirmed by the study where $90 \%$ of the vendors indicated that they had never been trained in hygiene.

According to the recommended conditions for hand washing, food handlers are required to wash their hands before starting work, after any absence from a work station, after blowing the nose or touching the face or hair, after use of toilet/latrine, after breaks/moments when off duty, after touching dirt/soil/debris/chemicals/any other dirty materials/surfaces, after performing maintenance works on equipments and after picking up objects from surfaces suspected to be contaminated [13]. Failure to observe these conditions could result into hazards from the vendors' hands entering the food or could facilitate the hazards to build up in the food. Defective hand washing can facilitate the transmission of pathogenic bacteria found in the environment and on people's hands via food to humans [14]. Handling food with unwashed hands may result in cross-contamination, 
hence introduction of bacteria on safe food [10]. Hand washing is important because human beings are the largest source of contamination to food [15]; therefore ready-to-eat food should not be handled with unwashed hands [16].

Table 1. Conformity to recommended conditions of Hand washing facilities.

\begin{tabular}{|c|c|c|c|c|c|}
\hline Observations & Scores & Number of recommended conditions & Najembe $(\%) \quad N=20$ & Lukaya (\%) N=21 & $\begin{array}{l}\text { Average \% for } \\
\text { Najembe and Lukaya }\end{array}$ \\
\hline Hand washing facilities & & 4 & & & \\
\hline High level conformity & 4 & & - & - & - \\
\hline Good conformity & 3 & & 2 & - & 1 \\
\hline Fair conformity & 2 & & 4 & 1 & 3 \\
\hline Low conformity & 1 & & 2 & 3 & 2 \\
\hline Non-conformity & 0 & & 92 & 96 & 94 \\
\hline
\end{tabular}

(4=High level conformity, $3=$ Good conformity, 2=Fair conformity, 1=Low conformity, 0 = non-conformity)

Although there appeared to be differences in the levels of conformity to recommended conditions for hand washing facilities at the stall in the two markets (Najembe and Lukaya) as presented in Table 1, the difference was not significant (Mann-Whitney U-test for significance - U>5).

\subsection{Conformity to Recommended Hygiene Conditions for Food Storage Equipments at the Stalls}

Forty two percent $(42 \%)$ of the stalls in the two markets studied (Lukaya and Najembe) scored 1 against the recommended conditions of food storage equipments; which denotes low conformity. Forty nine percent (49\%) scored 2 denoting fair conformity, and 9\% scored 3 denoting good conformity. None of the stalls scored 4 (Table 2). The fair to low level of conformity observed in the majority of the stalls was because most vendors used their stalls to prepare and store the meat or any other food items. A significant proportion of vendors interviewed (45\%) indicated that they were storing meats in boxes. Others $(43 \%)$ said that they were storing meats in buckets. They would then be positioned in the corners of the stalls where they were kept at ambient temperature. Ninety four percent (94\%) of the vendors interviewed indicated that they were keeping food stuffs at ambient temperature. This kind of storage made stalls fail to conform to recommended conditions of storage. Most storage equipments for meat vendors were observed to be dirty and improperly covered. They were not raised off the ground and meats were stacked high and deep in these equipments. Most of the equipments were not made of cleanable materials. They were exposed to dust raised by moving vehicles while in some, bad smell was coming from storage facilities which could have been a sign of high bacterial contamination.

Table 2. Conformity to recommended conditions of food storage equipments.

\begin{tabular}{|c|c|c|c|c|c|}
\hline Observations & Scores & Number of recommended conditions & Najembe (\%) $\mathbf{N}=\mathbf{2 0}$ & Lukaya $(\%) \mathrm{N}=21$ & $\begin{array}{l}\text { Average } \% \text { for } \\
\text { Najembe and Lukaya }\end{array}$ \\
\hline Food storage equipments & & 13 & & & \\
\hline High level conformity & 4 & & - & - & - \\
\hline Good conformity & 3 & & 10 & 9 & 9 \\
\hline Fair conformity & 2 & & 52 & 46 & 49 \\
\hline Low conformity & 1 & & 38 & 45 & 42 \\
\hline
\end{tabular}

(4=High level conformity, 3= Good conformity, 2=Fair conformity, 1=Low conformity, 0 = non-conformity)

The recommended conditions require that vendors of ready-to-eat food should prepare enough food for the day so that they can sell it all in the same day since most of them do not have good storage facilities. Poor storage of left-over food could promote the sale of stale food [16]. Food that is kept at ambient temperature has high chances of spoilage due to quick bacterial growth that could compromise its microbial quality and cause illness to consumers [5]. Failure to observe proper storage and handling practices during storage could introduce Staphylococcus aureus and Escherichia coli in food [17].

Although there seemed to be a difference in the levels of conformity to recommended conditions of food storage equipments at the stall in the two markets (Najembe and Lukaya) as presented in Table 2, the difference was not significant (Mann-Whitney U-test for significance - U>5).

\subsection{Conformity to Recommended Hygiene Conditions of Food Preparation and Processing Equipments at the Stall}

Overall, $22 \%$ of the stalls scored 3 denoting good conformity to recommended conditions of food preparation and processing equipments; regarding roasting stoves. Sixty six percent (66\%) of the stalls scored 2 denoting a fair conformity and $12 \%$ scored 1 denoting low conformity. None of the stalls scored 4. For roasting wire-meshes, $8 \%$ of the stalls scored 3 denoting good conformity, $31 \%$ scored 2 denoting fair conformity, $44 \%$ scored 1 denoting low conformity while $17 \%$ scored 0 denoting nonconformity to recommended conditions. None of the stalls scored 4 (Table 3). 
Table 3. Conformity to recommended conditions of food preparation and processing equipments.

\begin{tabular}{|c|c|c|c|c|c|}
\hline Observations & Scores & Number of recommended conditions & Najembe (\%) $\mathbf{N}=20$ & Lukaya (\%) N=21 & $\begin{array}{l}\text { Average \% for } \\
\text { Najembe and Lukaya }\end{array}$ \\
\hline Roasting stove & & 10 & & & \\
\hline High level conformity & 4 & & - & - & - \\
\hline Good conformity & 3 & & 14 & 30 & 22 \\
\hline Fair conformity & 2 & & 76 & 55 & 66 \\
\hline Low conformity & 1 & & 10 & 15 & 12 \\
\hline Non-conformity & 0 & & - & - & - \\
\hline Roasting wire mesh & & 7 & & & \\
\hline High level conformity & 4 & & - & - & - \\
\hline Good conformity & 3 & & 5 & 10 & 8 \\
\hline Fair conformity & 2 & & 33 & 30 & 31 \\
\hline Low conformity & 1 & & 48 & 40 & 44 \\
\hline Non-conformity & 0 & & 14 & 20 & 17 \\
\hline Cutting/chopping facilities & & 4 & & & \\
\hline High level conformity & 4 & & 15 & - & 8 \\
\hline Good conformity & 3 & & 45 & 24 & 34 \\
\hline Fair conformity & 2 & & 20 & 33 & 26 \\
\hline Low conformity & 1 & & 10 & 43 & 27 \\
\hline Non-conformity & 0 & & 10 & - & 5 \\
\hline Roasting sticks & & 1 & & & \\
\hline High level conformity & 4 & & 48 & 44 & 46 \\
\hline Good conformity & 3 & & - & - & - \\
\hline Fair conformity & 2 & & - & - & - \\
\hline Low conformity & 1 & & - & - & - \\
\hline Non-conformity & 0 & & 52 & 56 & 54 \\
\hline
\end{tabular}

(4=High level conformity, $3=$ Good conformity, $2=$ Fair conformity, $1=$ Low conformity, 0 = non-conformity)

The fair to low conformity observed for the roasting stoves and fair to non-conformity observed for roasting wire-meshes were because most of the meat roasting stoves and roasting wire-meshes were stained with fat/oil from the meat. This was seen on all the roasting stoves and wire-meshes observed during the assessment. In addition, the stoves and wiremeshes were made of hard to clean materials such as twisted metallic pieces. They were not well raised off the ground and there were some pieces of meat stuck in the wire-meshes which indicates that they were not cleaned regularly. Most of the stoves and wire meshes were in a bad state of repair. Vendors were seen to be visiting stalls that did not roast their meat types to re-heat the meat products which raised potential for cross-contamination. During interviews, $63 \%$ of the vendors admitted to roasting different types of meat products on their stoves at the same time. This exposed the meat products to cross-contamination. This practice was very common among the beef and goat meat vendors. Most beef and goat meat vendors were observed to smear chicken fat on their roasted meats for them to look more attractive to customers. This also has the potential to increase the risk of cross-contamination of the meats with any contaminants on the chicken. Cross-contamination is a serious risk in food vending due to inadequate knowledge on how to control it which puts the consumers of such foods at high risk of buying contaminated ready-to-eat food [18]. It is normally hard to trace the source of contamination during incidences where cross-contamination occurs as a result of intermixing products and criss-crossing of food vendors [19].

In the assessment of conformity of cutting/chopping facilities or items such as knives, machetes/pangas, chopping woods/boards in Lukaya and Najembe markets, $8 \%$ of the stalls assessed scored 4 denoting high level conformity to recommended conditions of cutting/chopping facilities, $43 \%$ scored 3 denoting good conformity, $26 \%$ scored 2 denoting fair conformity, $27 \%$ scored 1 denoting low conformity and $5 \%$ scored 0 denoting non-conformity (Table 3 ). The good to fair conformity observed in the majority of the stalls was boosted by knives and pangas which were generally clean at most of the stalls. This is one of the few areas where the highway meat vendors scored highly during the assessment of facilities. Stalls scored poorly on conformity assessment of chopping woods and boards because they were highly stained with meat particles. They were hard to clean and had deep dents all over the surface which could aid growth of bacteria. Chopping items have a potential to aid growth and multiplication of a number of bacteria such as Staphylococcus aureus, Salmonella spp and many other bacteria in the coliform group [20]. Failure to regularly change and sterilize food chopping items could cause cross contamination due to chopping of different foods from the same surface which can alter the microbial quality of food [21]. It is important that chopping items get properly cleaned after every use and are replaced if they get damaged especially when they develop dents and scratches. Food handlers could also use different chopping items for raw and 
ready-to-eat foods to mitigate this challenge [22].

Forty six percent $(46 \%)$ of the stalls scored 4 for roasting sticks denoting a high level of conformity to recommended conditions. The rest $(54 \%)$ of the stalls scored 0 denoting non-conformity to recommended conditions. The nonconformity observed at $54 \%$ of the stalls was due to the dirt which was visible on roasting sticks (Table 3). Some roasting sticks were poorly kept (some were placed on the dirty floors of stalls) after procurement from the meat suppliers. Roasting sticks are among food contact surfaces that are often ignored by meat vendors which have potential for crosscontamination. Haphazard handling of any food contact item might increase the risk of food contamination [23].

Although there appears to be a difference in the levels of conformity to recommended conditions of food preparation and processing equipments at the stall in the two markets (Najembe and Lukaya) as presented in Table 3, the difference was not significant (Mann-Whitney U-test for significance - U>5).

\subsection{Conformity to Recommended Hygiene Conditions of Facilities for Display and Service of Food}

Overall, (36\%) of the stalls scored 3 for display plat forms denoting a good level of conformity to recommended conditions. Thirty seven percent (37\%) scored 2 denoting a fair level of conformity while $27 \%$ scored 1 denoting low conformity. None of the stalls scored 4 (Table 4). The good to fair performance exhibited by display platforms in the majority of the meat stalls was boosted by the attractiveness of products that were on display which was mainly used as a marketing strategy by the highway vendors rather than contributing to reducing potential for contamination. The vendors aimed at making their products appear to be clean and good looking even though the rest of the surroundings were not hygienic. The same strategy was utilized for the display platforms. Vendors knew that customers buy from displays that appeared clean to observers, so they often cleaned in front of their stalls and this included the spaces along the road where vehicles which stop for passengers to buy the roasted meat park. The majority of the vendors displayed their meat products at ambient temperature which they re-warmed rather than reheat from time to time. Most (91\%) of the vendors interviewed indicated that they do this because sometimes they take long to sell the foods and (60\%) indicated that it took them 3-5 hours to sell all the prepared food.

Table 4. Conformity to recommended conditions of display and service of food.

\begin{tabular}{|c|c|c|c|c|c|}
\hline Observations & Scores & $\begin{array}{l}\text { Number of recommended } \\
\text { conditions }\end{array}$ & $\begin{array}{l}\text { Najembe (\%) } \\
\mathbf{N}=\mathbf{2 0}\end{array}$ & $\begin{array}{l}\text { Lukaya } \\
(\%) \mathrm{N}=\mathbf{2 1}\end{array}$ & $\begin{array}{l}\text { Average \% for } \\
\text { Najembe and Lukaya }\end{array}$ \\
\hline Display plat forms & & 4 & & & \\
\hline High level conformity & 4 & & - & - & - \\
\hline Good conformity & 3 & & 28 & 43 & 36 \\
\hline Fair conformity & 2 & & 41 & 33 & 37 \\
\hline Low conformity & 1 & & 31 & 24 & 27 \\
\hline Non-conformity & 0 & & - & - & - \\
\hline Packing materials & & 7 & & & \\
\hline High level conformity & 4 & & - & - & - \\
\hline Good conformity & 3 & & 19 & 20 & 20 \\
\hline Fair conformity & 2 & & 38 & 5 & 21 \\
\hline Low conformity & 1 & & 43 & 70 & 56 \\
\hline Non-conformity & 0 & & - & 5 & 3 \\
\hline $\begin{array}{l}\text { Display dressing (aprons, hair restraints, mouth } \\
\text { covers and beard covers) }\end{array}$ & & 3 & & & \\
\hline High level conformity & 4 & & 20 & 5 & 12 \\
\hline Good conformity & 3 & & 20 & 24 & 22 \\
\hline Fair conformity & 2 & & 5 & - & 3 \\
\hline Low conformity & 1 & & 45 & 57 & 51 \\
\hline Non-conformity & 0 & & 10 & 14 & 12 \\
\hline
\end{tabular}

(4=High level conformity, 3= Good conformity, 2=Far conformity, 1=Low conformity, 0 = non-conformity)

The Food and Agricultural Organisation and World Health Organisation (FAO/WHO) Codex Alimentarius Commission (CAC) Regional Guidelines for the Design of Control measures for vended foods in Africa of 1997 require that ready-to-eat foods intended for continuous serving should be protected from environmental contamination and kept at the holding temperature of $60^{\circ} \mathrm{C}$ or above. Any food that has been out of this temperature for more than 2 hours should not be sold or even reheated for sell. FAO/WHO recommend that food vendors should display food in warmers and that warmers should not be used for reheating food.

Twenty percent $(20 \%)$ of the stalls in both markets 
(Lukaya and Najembe) scored 3 for packing materials denoting good conformity to recommended conditions. Twenty one percent $(21 \%)$ scored 2 denoting fair conformity, $56 \%$ scored 1 denoting low conformity and 5\% scored 0 denoting non-conformity. None of the stalls scored 4 (Table 4). The fair to low conformity observed in majority of the stalls was due to poor hygienic conditions of packing materials. The packing items were made of weak materials such as paper and polythene bags which did not have handles. The utilization of popular white/transparent polythene bags involved use of bare hands to open them which exposed them to the risk of contamination. Some vendors blew air into the packing materials while opening them before use which could introduce bacteria from the air or the vendor's mouth.

The Food and Agricultural Organisation and World Health Organisation (FAO/WHO) Codex Alimentarius Commission (CAC) Regional Guidelines for the Design of Control measures for vended foods in Africa of 1997 require food grade packing materials to be used and that ready-to-eat foods should not be handled with bare hands. Clean tongs, forks, spoons or disposable gloves should be used when handling, serving or selling food. All crockery used should be clean and dry and not handled by touching the food contact surfaces. The FAO/WHO guidelines further indicate that vendors should never blow into plastic bags, wrappers or packages used for food. Food handlers should also avoid handling money. If this is unavoidable, the food handler should wash their hands after handling money and before handling food again. All unsold ready-to-eat food that cannot be properly preserved should be disposed off in a hygienic manner at the end of the day.

For display dressing gear (aprons, hair restraints, mouth covers and beard covers), $12 \%$ of the stalls scored 4 denoting high level conformity to recommended conditions for display and sell of vended food, $22 \%$ scored 3 denoting good conformity, $3 \%$ scored 2 denoting fair conformity, $51 \%$ scored 1 denoting low conformity and $12 \%$ scored 0 denoting non-conformity (Table 4). All vendors interviewed indicated that they did not have designated dressing rooms. Some $(40 \%)$ indicated that they stored their personal belongings in bags. Others (35\%) just hanged them on the wall of their stalls while $20 \%$ of the vendors stored theirs in boxes. This could explain why most of the stalls did not meet the recommended conditions for aprons/dressing during food display and service. Improper or dirty dressing by vendors could result in higher contamination of readyto-eat food [2].

Most of the vendors that were interviewed did not implement proper personal hygiene practices which affected both Najembe and Lukaya markets. For instance $73 \%$ of the meat vendors indicated that they did not use hair restraints while handling food. Sixty five percent (65\%) did not trim and clean their finger nails as required and $32 \%$ admitted to wearing jewellery on their fingers and arms while handling food. Fifty nine percent (59\%) did not cover their burns, wounds, sores or scabs on the hands or arms and $80 \%$ admitted that they handled food when they had a communicable disease such as running stomach, flue and cough. Vendors pointed out that they could not miss to vend meat whether they were seen or not as long as they would be able to walk or run. All vendors interviewed were not aware of anything wrong with eating, drinking, smoking or chewing in a food handling area. None of the vendors interviewed used disposable tissue when coughing or sneezing. The vendors indicated through interviews that such hygiene recommendations were too stringent for them and that they would still forget and cough or sneeze without tissue even if it was provided to them to use for free. All vendors interviewed handle money while serving customers. They all admitted that they talk over food while handling it. Sixty one percent $(61 \%)$ of the vendors admitted to testing salt using their tongues during meat roasting and $18 \%$ of the vendors blow air in the packing materials while serving customers.

This indicates that there was lack of awareness in personal hygiene practices among meat vendors in highway markets. Food handling personnel play an important role in ensuring food safety throughout the chain of food preparation [19]. Food borne outbreaks are generally due to poor personal hygiene of food vendors [2]. Observance of personal hygiene during food preparation is important because human beings are the largest sources of food contamination [15]. Ready-toeat food should not be handled with bare hands [16]. Poor personal hygiene could result in cross-contamination, hence leading to introduction of bacteria on safe food [10]. The person handling money should not handle food; this is because money is dirty and can contaminate safe food [10]. Observing personal hygiene is vital for any food establishment. Any food handler, who observes other forms of hygiene but not personal hygiene, will definitely contaminate food. Training is therefore required for the vendors of ready-to-eat foods in various aspects of personal hygiene in order to minimize health risks associated with failure to observe personal hygiene $[16,17]$.

Although there was a difference in the levels of conformity to recommended conditions of display and service of food in the two markets (Najembe and Lukaya) as presented in Table 4 , the difference was not significant (Mann-Whitney U-test for significance - $U>5$ ).

\subsection{Comparison of Hygiene Facilities for Different Vended Meats}

The overall mean score of hygiene facilities for all the three products (chicken, beef and goat meat) was 1.36. This was a low level of conformity to recommended conditions of hygiene facilities. Hygiene facilities at the stalls for goat meat in both markets had the highest mean score (1.48), followed by the facilities for beef meat stalls (1.37). The lowest mean score (1.23) was recorded in the hygiene facilities of chicken stalls. However, the difference observed in mean scores of facilities for goat meat, beef and chicken at Najembe and Lukaya markets, was not significant (ANOVA; $\mathrm{P}>0.05)$. 


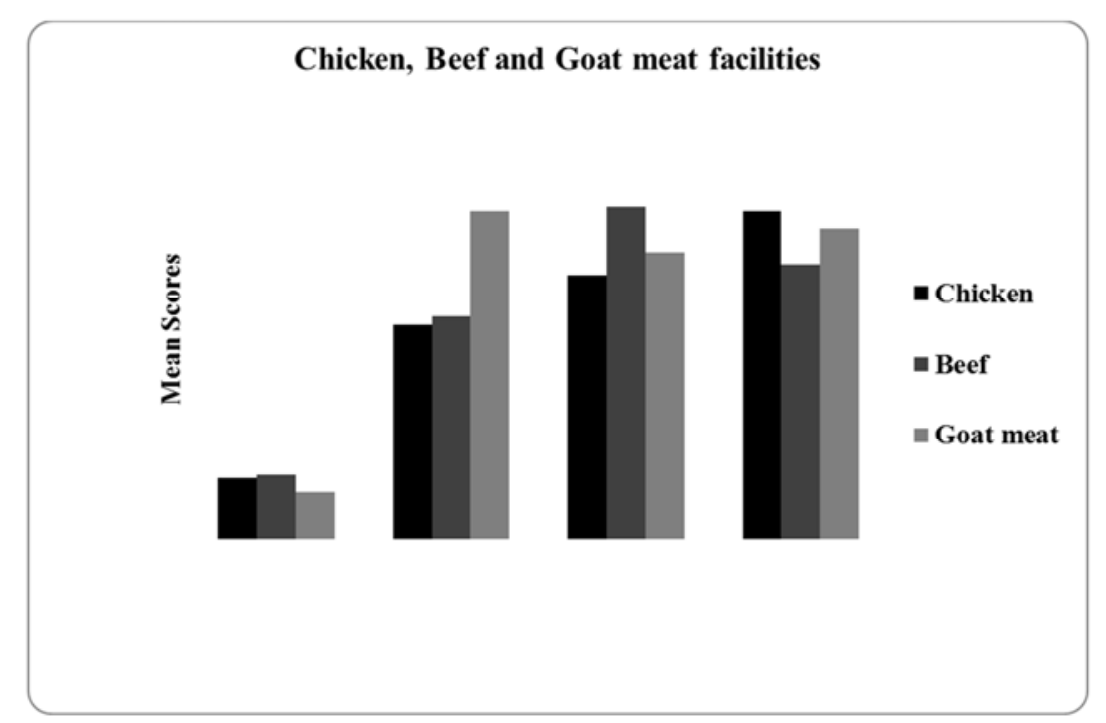

Figure 1. Mean scores obtained from the assessment of hygiene facilities for vended Chicken, Beef and Goat meat at Najembe and Lukaya markets.

For hand washing facilities, beef had the highest score (0.38) followed by chicken (0.36); goat meat had the lowest score $(0.28)$. The scores for hand washing facilities were affected by absence of hand washing materials/items such as well stocked small hand washing water containers (tippy taps) and detergents at most stalls particularly for goat meat. Concerning the food storage facilities, goat meat had the highest score (1.95), followed by beef (1.33) and chicken (1.28) in that order. The low scores of water facilities (especially in chicken) were mainly due to use of dirty storage items, use of storage equipments that are made of hard-to-clean materials, storage of food at ambient temperature and placing of storage equipments on dirty floors in the corners of stalls. For preparation and processing facilities, beef had the highest score (1.98), followed by goat meat (1.71) and chicken (1.57). Most of the food preparation equipments (especially for chicken) were observed to be dirty and stained, some were not made of cleanable materials while some were inadequate which explains the low scores obtained. For food display and service facilities, chicken had the highest score (1.95) closely followed by goat meat (1.85). Beef had the lowest scores (1.64). The scores for food display and service (beef in particular) were affected by use of packing materials that are not made of food grade materials, dirty and dressing materials such as aprons that were not fitting vendors and the absence of critical protective gear for vendors such as hair restraints and mouth pads which were lacking.

\section{Conclusions}

Overall, conformity of the hygiene facilities and practices for the meat products studied (chicken, beef and goat meat) was low for both markets. The low conformity across all the stalls in both markets is attributed to deficiencies in critical facilities such as food storage equipments, roasting stoves, cutting/chopping items, dressing gear and hand washing equipments. The low performance of hygiene practices is attributed to low awareness about good hygiene practices among the meat vendors. Also the low performance of both the facilities and practices indicates that monitoring by local authorities is not effective and does not promote vendors' compliance to recommended best practices.

The glaring problem of lack of hand washing facilities in both markets even at Lukaya which had just been renovated indicates the lack of priority by both government and market authorities about hygiene of food that is served in the markets. It also indicates that the market authorities and vendors are not aware of the importance of hand washing in securing the food that is served to customers. This has implication to safety of the foods that are sold in these markets. The poor storage infrastructure for meats facilitates the multiplication of bacteria especially due to failure in maintenance of temperature ranges. In addition, the poor state of food preparation and processing equipment raises potential for cross-contamination. All these too, have implications to the safety of meat products. The malpractices of purchasing meat products from unlicensed or unauthorized suppliers, sharing preparation and processing facilities, smearing chicken fat on roasted beef and goat meats, displaying of ready-to-eat meats at ambient temperature and failure to observe good personal hygiene practices expose the meat to contamination which puts the health of consumers at risk.

To address the above deficiencies and ensure safety of the products that are vended in the markets, vendors should have adequate and well secured hand washing containers and detergents at their stalls. They should minimise the possibility of having leftovers by preparing meat that is consumed on the same day. They should also regularly clean their roasting stoves and wire-meshes to prevent multiplication of bacteria. During meat roasting, the vendors should first prepare few batches of meat and sell them before they can roast others in order to minimise the time and 
temperature lag during display and service of ready-to-eat meat products. The market chairpersons and their committee members should install storage facilities from where vendors can temporarily store their products during roasting since most vendors cannot afford cold storage facilities.

The Department of Environmental Health and Food in the Ministry of Health together with the Department of Public Health and Marketing in the Ministry of Agriculture Animal Industry and Fisheries (MAAIF) and District Health Committees should partner with the market chairpersons and their committee members to gazette areas for hand washing facilities in strategic locations of the markets where all vendors can access. These authorities should also put in place mechanisms for training food vendors and market authorities in good food hygiene practices. A monitoring program should be put in place to enforce good hygiene practices.

\section{References}

[1] S. Sebudde, R. Kabagambe and M. Muganwa, "Hygiene and sanitation in public eating places in one municipal health system of Uganda, Kampala," Erudite Journal of Medicine and Medical Science Research (EJMMSR), Vol. 1, No. 1, 2012, pp. 1-8.

[2] G. Chirag, "Study of Hygienic practices of street food vendors in Allahabad city, India and Determination of Critical control points for safe street food," The Allahabad Farmer, Vol. 68, No. 2, 2013, pp. 88-106.

[3] C. Muyanja, L. Nayiga, B. Namugumya and G. Nasinyama, "Practices, knowledge and risk factors of street food vendors in Uganda," Food control, Vol. 22, No. 10, 2011, pp. 1551.

[4] A. Bagumire, M. Birungi, D. Bamwirire, W. Ssali, M. Dillon and S. Hannah, "Cleaning Guide for Fresh food Factories," A handbook for guiding food establishment workers and Sanitation managers. Published by NARO, 2000.

[5] P. Mensah, "Street Food in Accra, Ghana: how safe are they?." Bulletin of the World Health Organization, Vol. 80, No. 6, 2012, 546-556.

[6] NSW, "Food risk assessment of NSW food safety schemes," Food Authority-NSW Government, 2009.

[7] FDA, "Draft Methodological Approach to Identifying HighRisk Foods under Section 204 (d) (2) of the FSMA," 2013. http://www.fda.gov/Food/GuidanceRegulation/FSMA/ucm38 0210.htm.

[8] Ministry of Agriculture Animal Industry and Fisheries, "The manual of standard operating procedures for fish inspection and quality assurance," Department of Fisheries Resources, Entebbe Uganda, 2008.

[9] A. Bagumire, E. C. D. Todd, and G. W. Nasinyama and C. Muyanja, "Food Safety-related measures in the emerging aquaculture enterprises in Sub-Saharan Africa: Compliance of Uganda's operations against international market guidelines," African Journal of Food Science, Vol. 5, No. 22, 2010, pp. 920.
[10] FAO/WHO, "Regional guidelines for the design of control measures for street-vended foods (Africa) - Codex Alimentarius Supplement," Joint FAO/WHO food standards program, Agriculture and consumer protection department, FAO, Rome, 1997.

[11] Republic of Uganda, "The Food and Drugs Act" Cap 278 of Laws of Uganda, 2000.

[12] Republic of Uganda, "The Public Health Act of Uganda" Cap 281 of Laws of Uganda, 2000.

[13] A. Bagumire, "Food Hygiene for Food Business Operations: An elaborate Guide for Operators of hotels and restaurants, kitchens, milk centers, meat centers, food processing establishments, feed processing establishments, and farms," Food Safety Associates Guide Book Series 1. In press, 2014. http://foodsafetyltd.com/food-hygiene-and-food-safety.

[14] N. Barro, Bello A., Itsiembou Y., Sevadogo A and C. Ouattara, "Street-Vended Foods Improvement: Contamination Mechanisms and Application of Food Safety Objective Strategy: Critical Review," Journal of Nutrition, Vol.6, No.1, 2007, pp. 1-10.

[15] N. Marriot, "Principles of food sanitation," Van Nostrand Reinhold Company, New York. Vol. 1, No. 9, 1985, pp. 7080 .

[16] O. Muinde, "Hygienic and sanitary practices of vendors of street foods in Nairobi, Kenya," African Journal of Food Agriculture Nutrition and Development, Vol. 7, No. 4, 2005, pp. $1684-5378$.

[17] N. Odu, "Microbiological Quality of Street-Vended-ReadyTo-Eat "Bole" Fish In Port Harcourt Metropoplis," New York Science Journal, Vol. 6, No. 8, 2013, pp. 11-25.

[18] D. Tambekar, V. Jaiswal, D. Dhanorkar, P. Gulhane and M. Dudhane, "Identification of microbiological hazards and safety of ready-to-eat food vended streets of Amravati City, India," Journal of Applied Biosciences, Vol. 7, 2008, pp. 195 201.

[19] F. Bryan, P. Teufel, S. Riaz, S. Roohi, F. Qadar and Z. Malik, "Hazards and critical control points of street-vending operations in a mountain resort town in Pakistan," Journal of Food Protection, Vol. 55, No. 28, 1992, pp. 701-707.

[20] M. Annett, K. Rolf, W. Alfred and S. Kornelia, "Survival of bacteria on wood and plastic particles: Dependence on wood species and environmental conditions," Holzforschung, Vol. 59, 2005, pp. 72-81.

[21] S. H. Abrishami, B. D. Tall, T. J. Bruursema and P. S Epstein, "Bacterial adherence and viability on cutting board surfaces" Journal of Food safety, Vol. 14, No. 8, 1994, 153-172.

[22] Food Standards Agency, "Cleaning: Advice on keeping your hands, worktops and utensils clean in the kitchen," http://www.food.gov.uk/northernireland/nutritionni/niyoungpeople/survivorform/dontgetsick/cl eaning\#.U5cXSnbGa4k.

[23] C. R. Friedman, "Risk Factors for Sporadic Campylobacter Infection in the United States: A Case-Control Study in FoodNet Sites," Clinical Infectious Diseases, Vol. 38, No. 3, 2004, pp. 285-96. 